\title{
Molière actualisé par l'image dans les manuels de collège et de lycée d'aujourd'hui
}

A New Perception of Molière in Today's French Schools Text-Books

Isabelle Calleja-Roque

\section{OpenEdition}

\section{Journals}

Édition électronique

URL : http://journals.openedition.org/recherchestravaux/934

ISSN : 1969-6434

\section{Éditeur}

UGA Éditions/Université Grenoble Alpes

\section{Édition imprimée}

ISBN : 978-2-37747-014-3

ISSN : 0151-1874

\section{Référence électronique}

Isabelle Calleja-Roque, « Molière actualisé par l'image dans les manuels de collège et de lycée d'aujourd'hui », Recherches \& Travaux [En ligne], 91 | 2017, mis en ligne le 01 octobre 2017, consulté le 08 septembre 2020. URL : http://journals.openedition.org/recherchestravaux/934

Ce document a été généré automatiquement le 8 septembre 2020

(C) Recherches \& Travaux 


\title{
Molière actualisé par l'image dans les manuels de collège et de lycée d'aujourd'hui
}

\author{
A New Perception of Molière in Today's French Schools Text-Books
}

\author{
Isabelle Calleja-Roque
}

1 Molière est certainement encore aujourd'hui l'auteur patrimonial par excellence. Présent dans les programmes scolaires dès 1803 avec Le Misanthrope en classe de rhétorique, il n'a jamais cessé d'être étudié. Par ailleurs, les nombreuses représentations de ses pièces témoignent de sa fortune littéraire : l'actualité théâtrale de la Comédie-Française affiche chaque saison au moins un titre de l'auteur. En 1978, avec le film d'Ariane Mnouchkine, Philippe Caubère jouant le rôle de Molière, le cinéma s'empare de l'œuvre du dramaturge. Depuis lors, la vie et l'œuvre de Molière ont été plusieurs fois portées à l'écran (adaptation de L'Avare réalisée en 1981 par Jean Girault, avec Louis de Funès dans le rôle d'Harpagon; ou encore, autre exemple, Molière de Laurent Tirard en 2007, avec Romain Duris).

2 Toutes les mises en scène théâtrales des comédies de Molière et tous les films réalisés sur la vie de l'auteur-acteur actualisent à la fois l'image du dramaturge et celle de son œuvre. Ils fournissent ainsi aux auteurs et aux éditeurs des manuels d'aujourd'hui une importante réserve dans laquelle il est possible de puiser une iconographie riche en représentations de l'auteur et de son théâtre, pouvant favoriser à la fois leur contextualisation et leur actualisation.

3 C'est pourquoi il m'a paru intéressant de m'interroger ici sur les rapports que les manuels $d u x^{e} I^{e}$ siècle $d u$ collège et du lycée ont établis avec cette dynamique de l'actualisation-contextualisation de Molière et de son œuvre. En quoi ce double mouvement oriente-t-il la lecture scolaire de l'image du dramaturge et de ses comédies? Permet-il de faire réfléchir les élèves sur la relativité des sens des œuvres dont les enjeux multiples ont contribué, au fil des époques, à orienter l'interprétation vers une pluralité de lectures, en particulier sociales, politiques, ou symboliques? Mon propos envisagera l'image de l'auteur avant l'analyse de ses comédies, à partir d'un 
corpus de treize manuels (sept pour le collège et six pour le lycée) publiés par cinq éditeurs différents (Belin, Didier, Hachette, Hatier, Magnard) entre les années 2007 et 2016.

\section{Molière, une image de l'auteur actualisée de manière étonnante}

4 Une première remarque s'impose: dans les manuels contemporains, l'image de Molière, en tant qu'auteur, semble avoir un statut particulier, qui différencie la représentation du dramaturge de celle de tout autre auteur patrimonial.

Pour les auteurs patrimoniaux des siècles passés, l'image donnée à voir aux élèves passe souvent par une iconographie contextualisante : portraits d'époque ou gravures. C'est, bien entendu, le cas pour Molière. Pour l'image du dramaturge, les manuels ont ainsi recours aux différents portraits des frères Mignard, qui ont représenté l'auteur de son vivant. Ainsi, l'image de l'auteur-acteur donnée à voir aux élèves est celle d'un Molière jeune et romantique : longue perruque frisée, regard qui exprime un air mélancolique, petite moustache, lèvres charnues (fig. 1). Cette contextualisation de l'image de l'auteur est redoublée, de façon tout à fait courante dans les manuels d'aujourd'hui, par une seconde iconographie : la gravure de Claude Simonin, datant, elle-aussi, du vivant de Molière, intitulée Le vray portrait de Mr de Molière, laquelle le représente en habit de Sganarelle (fig. 2).

Figure 1. - Mignard Nicolas, Portrait de Molière dans le rôle de César, dans La Mort de Pompée, xvıle siècle.

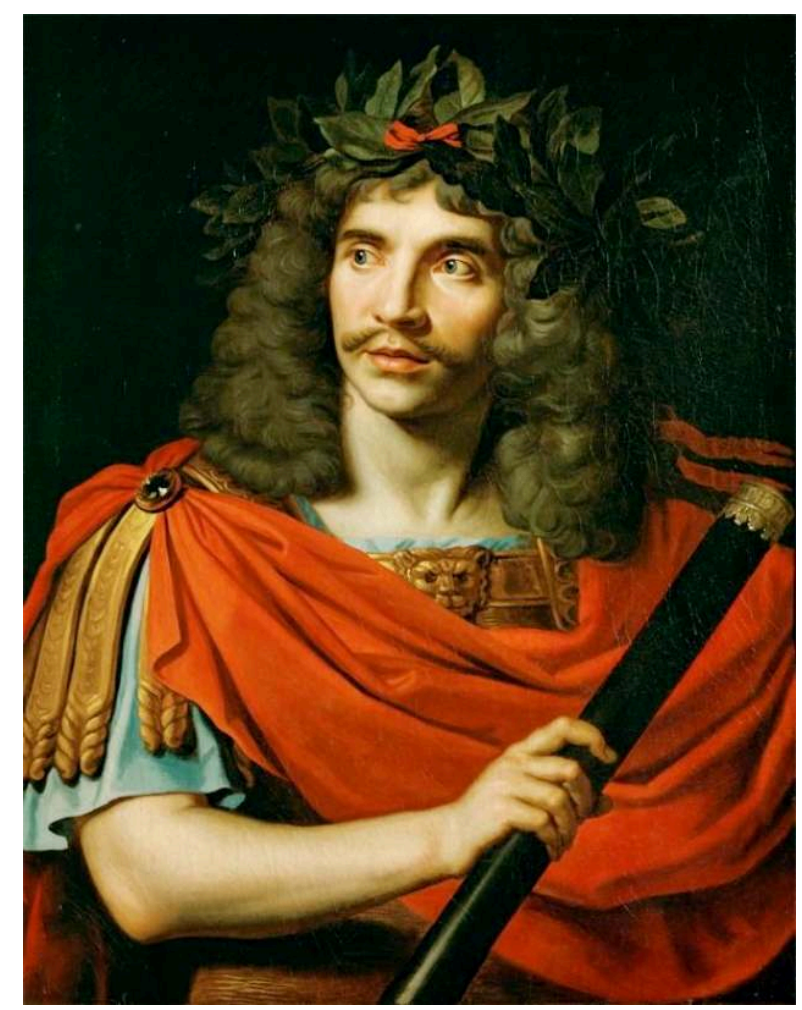

Huile sur toile, Bibliothèque de la Comédie-Française, Paris. 
Figure 2. - Simonin Claude, Le Vray portrait de Mr de Molière en habit de Sganarelle, xvıl ${ }^{\mathrm{e}}$ siècle.

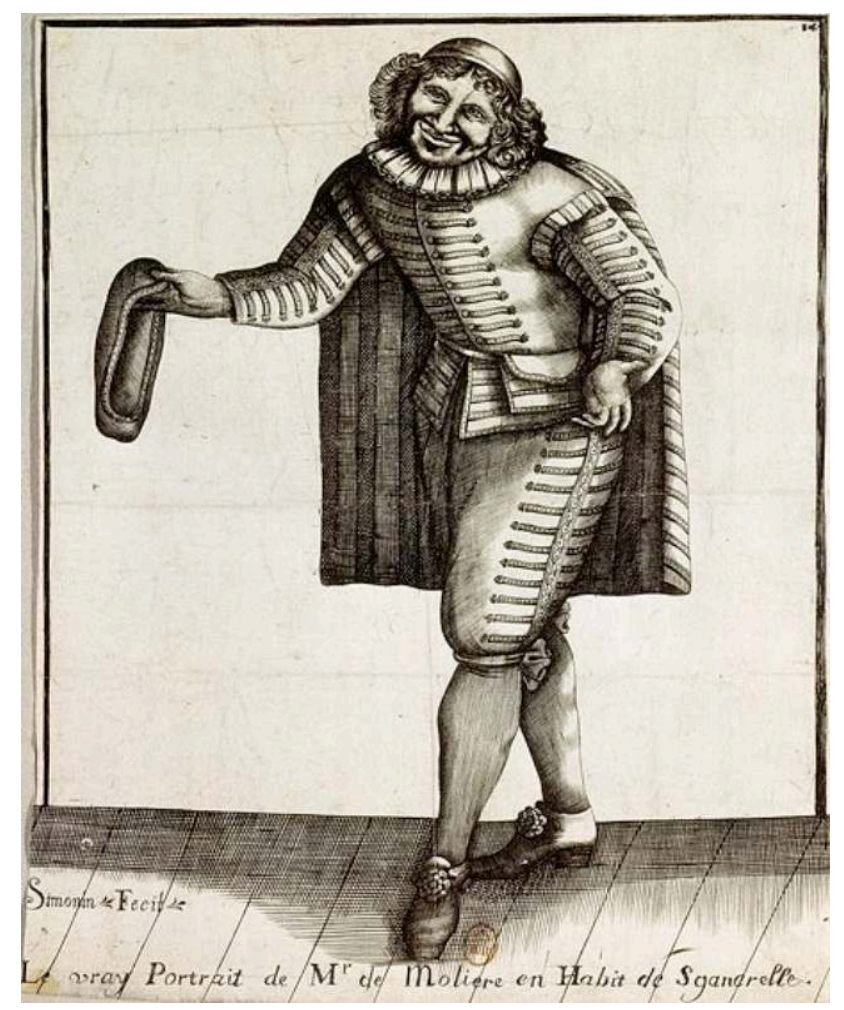

Gravure, Bibliothèque nationale de France.

6 Ainsi, dans les manuels, deux images de Molière coexistent : d'un côté celle de l'écrivain qui est celle d'un homme dont la caractéristique principale est la noblesse des traits et, d'un autre côté, celle du comédien que l'on donne à voir grimaçant dans le rôle du valet Sganarelle.

Cependant, ce qui est unique dans le cas de Molière, c'est que les manuels d'aujourd'hui juxtaposent deux représentations contextualisantes et une iconographie qui actualise l'image du dramaturge. Il s'agit d'images fictionnelles de l'auteur qui se superposent aux portraits précédents. Ces images sont celles des cinéastes qui ont réalisé des biographies cinématographiques de Molière, en particulier Ariane Mnouchkine, en 1978, et Laurent Tirard, en 2007. Pour les élèves d'aujourd'hui, le visage de Molière est donc actualisé à travers celui de Philippe Caubère ou de Romain Duris, tels qu'ils apparaissent sur les affiches des deux films, rappelant étroitement les traits de l'homme représenté sur les toiles des frères Mignard.

Il faut interroger cette actualisation de l'image de l'auteur, car elle oriente nécessairement la lecture de l'élève. Ainsi, il est intéressant de noter que, pour le film d'Ariane Mnouchkine, deux affiches coexistent. Or, une seule d'entre elles est reprise dans les manuels. Il s'agit de celle où l'on voit Molière les cheveux au vent, la bouche ouverte, pris comme sur le vif en plein combat contre ses opposants (fig. 3). Il porte la longue perruque frisée et la fine moustache caractéristiques des représentations des frères Mignard. Des personnages de son théâtre sont représentés en mouvement en bas de l'affiche, donnant la sensation d'une fête perpétuelle. Le tout baigne dans des couleurs vives qui accentuent l'impression de force et de détermination qui émane du visage du comédien. 
Figure 3. - Affiche ${ }^{\circ} 1$ du Molière d'Ariane Mnouchkine (1978).

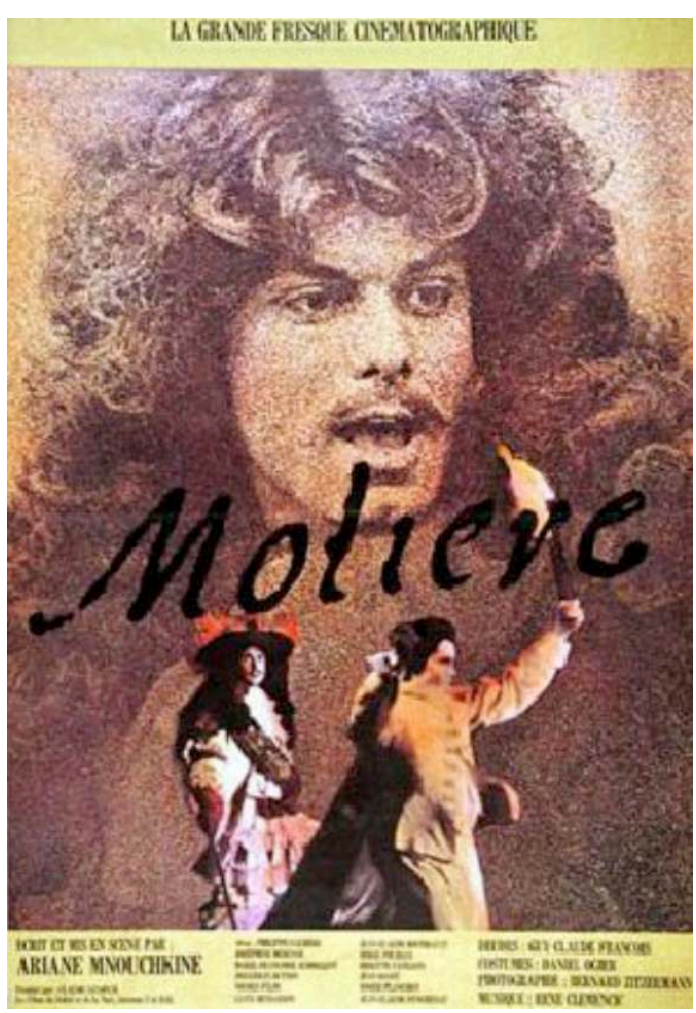

9 À l'inverse, la seconde affiche, beaucoup plus sombre dans les couleurs, représente Molière les cheveux plus courts, sans perruque, coiffé d'un vaste chapeau. Le regard est fixe, les yeux sont écarquillés. Il semble contempler son propre destin, lequel est figuré en bas de l'affiche de façon tragique par un personnage qui évoque la mort (fig. 4). Le fait que cette deuxième affiche ne soit jamais présente dans les manuels est révélateur de l'image que les ouvrages scolaires d'aujourd'hui choisissent de donner à voir en l'actualisant par le recours à l'iconographie fictionnelle : c'est celle d'un homme libre, passionné et combatif qui a voué sa vie au théâtre et dont le parcours est donné en exemple aux élèves. 
Figure 4. - Affiche $n^{\circ} 2$ du Molière d'Ariane Mnouchkine.

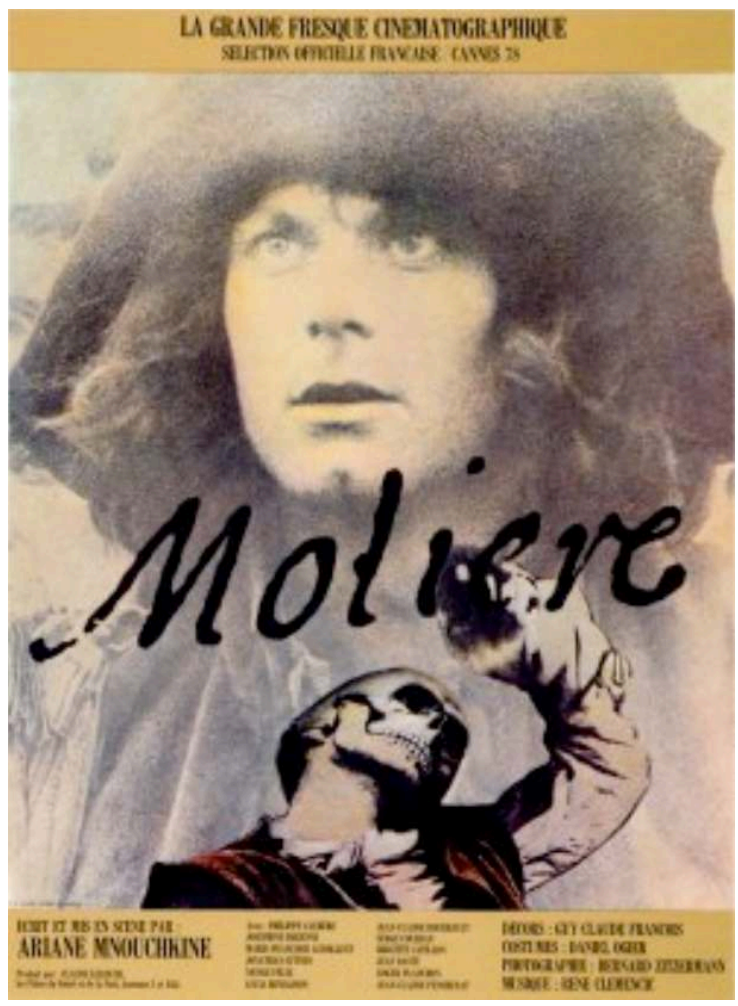

10 On reconnaît la même symbolique dans l'affiche du film de Laurent Tirard. Molière y est représenté les cheveux au vent, courant dans un champ de coquelicots. Il porte un sac de voyage sur le dos et semble vouloir affronter son destin avec beaucoup de détermination (fig. 5). 
Figure 5. - Affiche du Molière de Laurent Tirard (2007).

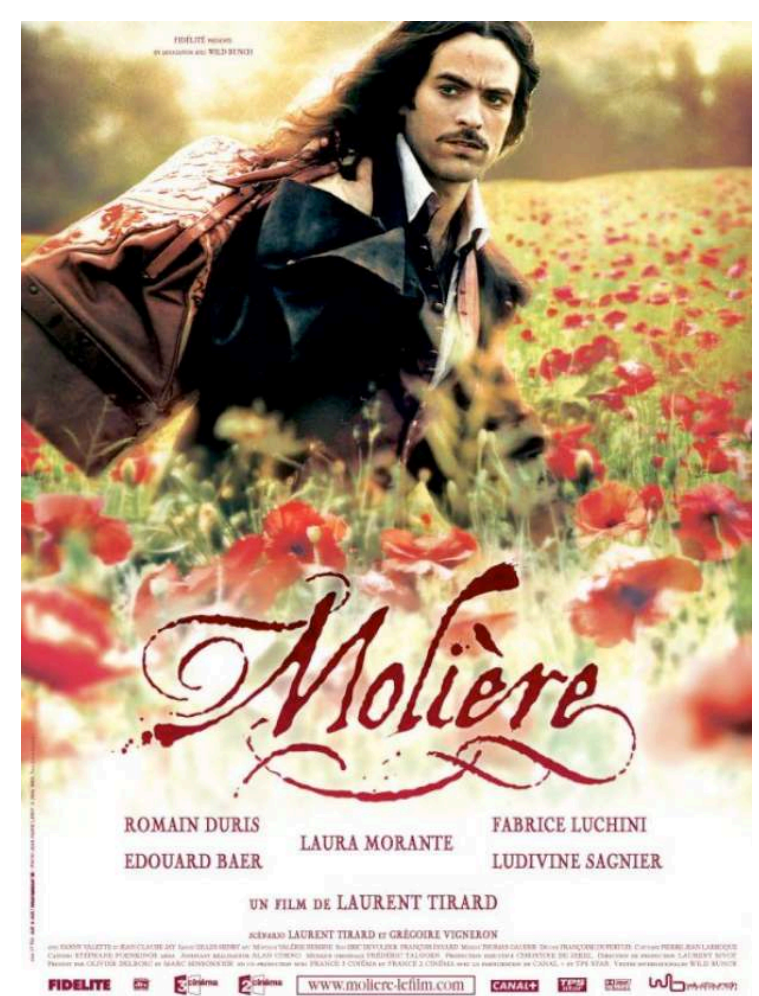

11 Dans le manuel de 2011 pour la classe de $5^{\mathrm{e}}$ des éditions Didier, Fil d'Ariane, on retrouve ainsi l'affiche du film d'Ariane Mnouchkine en pleine page, en ouverture du chapitre consacré à l'étude de la comédie ${ }^{1}$. Un peu plus loin, c'est l'affiche du film de Laurent Tirard qui donne lieu à une analyse de l'image, laquelle invite l'élève à identifier dans la figuration de Molière l'image d'un homme « à la fois libre et déterminé ${ }^{2}$ ».

Certains manuels utilisent même la dynamique de la contextualisation-actualisation pour orienter l'interprétation de l'élève, en faisant le choix de juxtaposer une double représentation de la figure de l'auteur. C'est le cas, par exemple, du manuel de 2007, pour la classe de $4^{\mathrm{e}}$ des éditions Belin, dans lequel on peut voir, sous le portait de Nicolas Mignard peignant Molière dans le rôle de César, celui de Philippe Caubère jouant Molière dans le film d'Ariane Mnouchkine ${ }^{3}$. La superposition de ces deux iconographies produit certainement pour l'élève un effet d'identification de l'image de l'auteur à la représentation actuelle donnée par le comédien l'incarnant dans le film d'Ariane Mnouchkine. Mais elle peut aussi lui permettre de percevoir le double jeu de l'actualisation-contextualisation. Lorsque l'image de l'auteur est contextualisée par la représentation du portrait de Molière jouant dans La Mort de Pompée de Nicolas Mignard et par la gravure de Claude Simonin représentant Molière en Sganarelle, tout comme, lorsque cette image de l'auteur est actualisée par l'introduction dans les manuels des photographies de l'affiche du film d'Ariane Mnouchkine et de celui de Laurent Tirard, ce double geste de contextualisation-actualisation a nécessairement des incidences sur l'interprétation de la figure de l'auteur qui est donnée à lire aux élèves. Dans tous les manuels d'aujourd'hui, il s'agit de donner à voir l'image d'un Molière en tant qu'auteuracteur qui a voué sa vie au théâtre, un homme qui a été avant tout un grand comédien.

C'est certainement l'importance de la place du théâtre comme art du spectacle dans les programmes de 2001 de l'enseignement secondaire, en lieu et place du théâtre 
essentiellement conçu à partir du texte comme c'était le cas précédemment, qui explique cette présentation de Molière dans les ouvrages du collège et du lycée. Celle-ci émane, comme nous allons le voir maintenant, de la présence dans les manuels d'une double iconographie dont l'objectif est à la fois de contextualiser et d'actualiser l'image du dramaturge.

\section{Entre contextualisation et actualisation de la comédie moliéresque, une porte ouverte vers la relativité du sens des œuvres}

Les manuels sont largement soucieux d'inscrire l'œuvre de Molière dans le contexte de sa production. C'est en général dans le cadre de la transmission des savoirs savants sur la découverte du théatre classique ainsi que de l'époque de l'auteur que se situent la plupart des éléments de cette contextualisation. Nombreux sont les ouvrages du collège qui, en lien avec l'histoire des arts, offrent une riche iconographie dans ce domaine, afin de faire découvrir aux élèves le statut d'acteur de Molière et d'auteur en lien avec le mécénat royal. Ainsi, par exemple, de façon tout à fait inventive et originale, Jardin des lettres, manuel de 2011 des éditions Magnard pour la classe de $5^{\mathrm{e}}$, propose un atelier ludique conçu sous la forme d'un jeu de l'oie intitulé Le roi et le comédien. L'objectif visé relève de l'histoire littéraire et consiste à comprendre comment un artiste tel que Molière pouvait accéder à la célébrité au temps du Roi-Soleil ${ }^{4}$. Au travers du jeu, constitué d'un plateau de 43 cases, il s'agit, pour les élèves, de s'identifier à Molière en parcourant les différentes étapes auxquelles est associée une iconographie contextualisante apportant des informations culturelles sur la vie du dramaturge et sur les divertissements à la cour de Louis XIV. On peut retrouver, en particulier, dans les 43 cases, le fameux fauteuil de Molière, différentes représentations du dramaturge, le portrait de Louis XIV, des photos de Versailles et de son jardin, ainsi que les frontispices de quelques pièces ou encore des gravures d'époque illustrant plusieurs des comédies de l'auteur. Au terme du jeu, l'équipe gagnante est celle qui parvient la première sur la dernière case et qui, grâce aux informations récoltées, est capable de faire un résumé complet de la vie de Molière. De façon ludique, ce manuel apporte donc aux élèves de précieux documents susceptibles de les aider à contextualiser à la fois la vie de l'écrivain ainsi que les rapports entre les artistes et le pouvoir au XvII ${ }^{\mathrm{e}}$ siècle.

On retrouve des éléments similaires de contextualisation dans les manuels du lycée, mais de manière sérieuse cette fois, sous la forme de fiches techniques présentant la vie de Molière ou/et l'évolution de la comédie depuis l'Antiquité jusqu'au XVII ${ }^{e}$ siècle 5 . Dans ces ouvrages, on constate, comme c'est le cas dans le manuel de 2010 pour la classe de $2^{\text {nde }}$ des éditions Belin ${ }^{6}$, la présence de frontispices des comédies de Molière, quelques gravures d'époque ainsi que la reproduction du tableau d'Ingres, Louis XIV et Molière à table devant les courtisans ${ }^{7}$. Il semble donc que, en ce qui concerne le contexte de la vie de l'auteur, les manuels du collège comme ceux du lycée soient soucieux d'apporter aux élèves suffisamment de documents.

En ce qui concerne l'actualisation, des photographies du film d'Ariane Mnouchkine jouent souvent ce rôle, en particulier dans les ouvrages du collège, à propos de la représentation de la vie de Molière, comme on peut le constater dans le manuel de 2011 des éditions Belin pour la classe de $5^{\mathrm{e}}$, L'œeil et la plume $e^{8}$, ou encore dans celui publié par 
les éditions Magnard pour la classe de $6^{\mathrm{e}}$, Jardin des lettres ${ }^{9}$. Dans ce dernier ouvrage, une double page invite l'élève à découvrir la vie de Molière en suivant le film de la cinéaste à travers une série de photos. Dans ce cas, l'actualisation se double d'un mouvement de contextualisation: on permet à l'élève d'actualiser le contexte de la production de l'œuvre molièresque dans une approche qui lui permet de concrétiser le passé à travers des images d'aujourd'hui.

De même, les ouvrages scolaires se montrent particulièrement soucieux de faire vivre dans l'ici et le maintenant le texte de Molière. Pour actualiser sa réception, on a recours aux mises en scène les plus récentes. Dans un perpétuel souci de rapprocher la comédie moliéresque du vécu des élèves, on n'hésite pas à faire appel à l'imaginaire des metteurs en scène les plus créatifs qui transposent par exemple, comme le fait Jean-Luc Moreau en 1994, Les Fourberies de Scapin dans un décor où échafaudages, jerricans d'essence et chaîne stéréo campent un univers contemporain. Des illustrations de cette mise en scène se trouvent dans de nombreux manuels de la classe de $5^{\mathrm{e}}$ depuis le début du xxI ${ }^{e}$ siècle. Toujours dans le même souci d'actualisation, Fil d'Ariane, par exemple, propose en complément de deux photographies de cette mise en scène, un extrait du début de la farce Ahmed le Subtil ou Scapin 84, texte écrit en 1984 par Alain Badiou qui transpose la comédie de Molière dans l'univers des banlieues. On peut constater que le questionnaire qui accompagne ces documents vise bien l'actualisation de la comédie de Molière. En effet, on demande aux élèves de relever dans le texte les indications qui montrent que la pièce d'Alain Badiou est contemporaine et d'indiquer celles qui montrent comment le metteur en scène a « actualisé » la comédie. Il s'agit donc de faire des repérages, lesquels consistent à relever, dans le texte, les changements (personnages, lieux, situations...) qu'a opérés Alain Badiou; et dans les deux photographies, à observer la scénographie (décor et costumes). Cette double analyse débouche sur un exercice écrit intitulé "Actualisez Les Fourberies de Scapin ». L'élève doit trouver un nouveau titre à la pièce d'Alain Badiou, dresser une nouvelle liste des personnages en modernisant leur prénom et leur origine sociale, puis, "rédiger la didascalie initiale en situant l'action dans un lieu qui convient à notre époque ${ }^{10} »$. Pour les auteurs de ce manuel, "actualiser ", c'est donc rendre contemporain le texte de Molière, en l'inscrivant dans un contexte d'aujourd'hui. En modernisant la présentation des personnages, l'élève est amené à porter sur aujourd'hui un regard qui l'invite à se représenter le texte à l'aune de l'époque de Molière. On s'approche ainsi des lectures actualisantes définies par Yves Citton.

Les manuels du lycée proposent aussi une riche iconographie donnant à voir de nombreuses images de mises en scène actuelles des comédies de Molière. En règle générale, l'appareil didactique exploite cette iconographie dans le cadre de l'histoire des arts. Le questionnement repose surtout sur la double dimension iconique et iconographique par l'observation des costumes et des postures des comédiens. Plus rarement, le travail sur la mise en scène invite l'élève à comparer plusieurs propositions artistiques, comme c'est le cas pour Tartuffe dans le manuel de 2015 pour la classe de $2^{\text {nde }}$ des éditions Belin ${ }^{11}$, lequel accorde une double page à la mise en scène de la pièce. La confrontation entre Elmire et Tartuffe (acte IV, scène 5) y est montrée à travers quatre interprétations différentes, pour lesquelles l'appareil didactique précise chaque fois la démarche du metteur en scène (même si c'est de manière rapide) : « une vision majestueuse » renvoie à la mise en scène de Jacques Weber, en 1994, au théâtre Antoine; "une transposition orientale » donne à voir la mise en scène d'Ariane Mnouchkine au Festival d'Avignon, en 1995 ; «une proposition moderne» sert de 
légende à la mise en scène de Stéphane Braunschweig, en 2008, au théâtre de l'Odéon ; " une adaptation atemporelle » définit la mise en scène de Galin Stoev, en 2014, à la Comédie-Française. Les choix artistiques des quatre metteurs en scène sont analysés à travers l'observation des costumes, des décors, des accessoires, pour en relever les différences et les ressemblances.

La confrontation entre ces quatre interprétations du Tartuffe donne ensuite lieu à une réflexion sur la représentation des personnages dans chaque mise en scène, afin de permettre aux élèves de comprendre pourquoi et comment chaque artiste donne sens à la pièce en l'interprétant. Cette réflexion débouche sur un double exercice: on demande d'abord à l'élève de rédiger un paragraphe, lequel prend pour support les images afin de l'aider à s'interroger sur le fait que la représentation d'une pièce est toujours une interprétation du texte; on invite ensuite l'élève à proposer des idées pour la mise en scène d'un extrait du texte : costumes, décors et accessoires. Par ses choix iconographiques, cette proposition des auteurs du manuel est riche en enseignements pour les élèves. La juxtaposition des différentes photographies permet d'inscrire le texte de Molière dans l'ici et le maintenant. Les précisions apportées par l'appareil didactique ancrent le théatre dans sa double dimension : à la fois un genre littéraire et un spectacle vivant, lequel est la représentation jouée du texte écrit par un artiste d'aujourd'hui qui imprègne de son propre univers l'œuvre initiale. Ainsi, avec la transposition du Tartuffe dans un contexte oriental, la photographie du spectacle d'Ariane Mnouchkine est une porte ouverte vers une « lecture actualisante » de l'œuvre de Molière, telle que la définit Yves Citton. Comme celui-ci le souligne, l'exploitation de cette mise en scène peut permettre à l'enseignant de montrer qu' "un texte littéraire ne continue à exister que pour autant qu'il nous parle, qu'il ne nous parle que par rapport à nos pertinences actuelles ${ }^{12} »$. Ces quatre images permettent à l'élève de comprendre qu'un metteur en scène construit une lecture particulière, datée, située, de l'œuvre de Molière. Leur confrontation l'aide à prendre conscience que les grandes mises en scène jalonnent l'histoire des œuvres et en multiplient les sens. L'élève peut aussi, à partir de l'analyse de ces différentes propositions de metteurs en scène, construire son interprétation du Tartuffe en fonction de sa propre sensibilité, en y projetant ses propres connaissances, ses propres préoccupations et sa propre représentation du monde. Mais l'élève n'est qu'un sujet lecteur en apprentissage, sa lecture personnelle se situant, bien entendu, à un autre niveau que celle qu'a produite un metteur en scène. Pourtant, l'exemple de ces expériences théâtrales peut lui permettre de faire de la rencontre avec l'œuvre de Molière une occasion pour réfléchir sur soi et sur le monde qui nous entoure. L'appareil didactique du manuel ne va pas jusque-là cependant. Méconnaissant le paradigme du sujet lecteur, il n'invite pas l'élève à explorer par lui-même les possibles interprétations de la lecture du Tartuffe que propose chaque mise en scène. Cependant, même si, dans cette démarche, l'élève manque d'autonomie, l'interprétation actualisante de chaque mise en scène étant donnée en regard des photographies, il n'en demeure pas moins que cette double page vise à ancrer la lecture du texte de Molière dans de possibles significations a posteriori. À partir de ce support iconographique, l'enseignant est libre de poursuivre la démarche pour faire en sorte que l'élève, à son tour, se projette dans l'œuvre, et qu'il l'investisse en fonction de son propre univers mental pour en construire une lecture actualisante.

On notera que quelques manuels, du collège comme du lycée, permettent aujourd'hui cette démarche. L'étude du théâtre de Molière se trouve facilitée par l'abondance des supports iconographiques de mises en scène des comédies du dramaturge. En 2007, par 
exemple, Soleils d'encre, ouvrage pour la classe de $1^{\text {re }}$, propose un exercice de dissertation intéressant ${ }^{13}$. À la suite de l'étude de George Dandin, il s'agit de s'interroger sur l'actualisation du théâtre classique, à partir de propos de Roger Planchon extraits du programme pour la mise en scène de la pièce réalisée à Villeurbanne en 1987 : « Nous ${ }^{14}$ aimons cette pièce car elle a été écrite le mois dernier, c'est pour nous la définition d'un classique. » À l'appui de cette citation, plusieurs photographies de mises en scène de la pièce sont données à voir. Des pistes sont proposées pour aider à construire la réflexion et pour que les élèves puissent prendre conscience du fait que l'époque contemporaine peut se retrouver dans le théâtre classique. L'exemple d'Ariane Mnouchkine qui place Tartuffe dans une Algérie où "sévissent les islamistes » est évoqué. On invite aussi les élèves à s'interroger sur ce que peuvent nous dire de notre monde contemporain les pièces de Molière: «Les fractures sociales et les mariages forcés sont-ils totalement oubliés au $\mathrm{XxI}^{\mathrm{e}}$ siècle?»

21 Dès lors que, par la confrontation de photographies de différentes mises en scène, on a quelque peu " actualisé » la comédie de Molière dans une approche didactique qui tient compte de l'univers des élèves, que fait-on pour amener ces derniers à inscrire cette lecture en rapport avec les contextes originaux de production du texte et de sa réception première? Là encore deux exemples, choisis parmi les plus représentatifs, vont nous permettre d'apporter des éléments de réponse.

Depuis le début du xxi siècle, les manuels du collège proposent une réflexion sur la relativité du sens de l'œuvre de Molière. Nous l'avons dit, ils se montrent soucieux de conduire l'élève à percevoir que la lecture de ses comédies, suivant les époques, a pu être orientée différemment. Pour favoriser cette prise de recul, en complément des photographies de mises en scène actuelles, ils fournissent d'autres supports iconographiques. C'est le cas, par exemple, du manuel de 2007 pour la classe de $4^{\mathrm{e}}$ des éditions Belin, à l'occasion de l'étude de L'Avare en œuvre intégrale ${ }^{15}$. Ce manuel demande aux élèves de comparer trois interprétations du personnage d'Harpagon à des époques différentes: celle de l'acteur Grandmesnil, au XIX siècle; celle de Louis de Funès dans le film de Jean Girault en 1979; et celle de Michel Serrault dans le téléfilm de Christian de Chalonge en 2007: ce questionnement conduit l'élève à réfléchir à la complexité du personnage d'Harpagon et lui permet de comprendre que, selon les époques et les choix artistiques des metteurs en scène, la lecture du personnage peut être différente. Elle demeure cependant encore partielle car non contextualisante : elle ne conduit pas l'élève à inscrire cette lecture en rapport avec les contextes originaux de production du texte et de sa réception première.

En 2011, toujours pour la classe de $4^{e}$, l'ouvrage de la même édition approfondit quelque peu cette réflexion. Il propose d'entrer par l'image dans l'étude de L'Avare. Les auteurs du manuel donnent à voir le frontispice de la pièce, lequel aide l'élève dans cette démarche ${ }^{16}$. Des illustrations du xix ${ }^{e}$ siècle, une photographie d'Henri Virlojeux dans le téléfilm de Jean Pignol en 1978 et une autre de la mise en scène de Catherine Hiegel à la Comédie-Française en 2009, amènent l'élève à s'interroger sur l'évolution de la représentation du personnage, laquelle est synthétisée dans une rubrique intitulée : «Du comique au tragique ${ }^{17}$ ». Harpagon y est présenté comme un personnage «tout en contrastes qui a été interprété de façons très diverses ». On rappelle que certains metteurs en scène ont souligné sa folie et « son enfermement dans une obsession qui ne laisse place à rien d'autre »; alors que d'autres ont insisté sur " les effets désastreux de sa tyrannie sur l'ensemble de la famille »; et que d'autres enfin, comme dernièrement 
Catherine Hiegel, ont cherché à montrer son «caractère grotesque et la dimension farcesque de la pièce ". Il y a donc encore réflexion sur les évolutions du sens de l'œuvre même si la contextualisation reste très limitée. Cette démarche pédagogique, qui amène l'élève à comparer différentes mises en scène historiques des œuvres est cependant quasiment absente des manuels de lycée. C'est, en effet, uniquement à la lumière de la mise en scène de Catherine Hiegel que L'écume des lettres, manuel de 2011 pour la classe de $2^{\text {nde }}$ des éditions Hachette, propose de lire L'Avare ${ }^{18}$ : on peut émettre l'hypothèse que cette différence s'explique par l'orientation des instructions officielles du second cycle, lesquelles ont davantage tendance à favoriser des mises en scène contemporaines plutôt qu'une contextualisation du texte théâtral. En effet, pour l'étude du théâtre en classe de $1^{\text {re }}$, l'intitulé de l'objet d'étude est « le texte théâtral et sa représentation, du $\mathrm{XVII}^{\mathrm{e}}$ siècle à nos jours ${ }^{19}$ ». Il s'agit, entre autres choses, de "sensibiliser les élèves à l'art de la mise en scène, notamment dans sa capacité à enrichir l'interprétation ». Les enseignants sont, pour ce faire, invités à prendre appui sur une "programmation locale ou sur des captations ». Cette dernière préconisation pourrait expliquer le choix des auteurs des manuels de lycée, en vue de la préparation aux épreuves du baccalauréat, de privilégier dans leurs pages une approche centrée sur des images de mises en scène d'aujourd'hui.

On peut reconnaître, en conclusion, l'effort des auteurs des manuels du collège et du lycée pour inscrire Molière et de son théâtre dans une double démarche de contextualisation-actualisation. Même si ces tentatives ne sont pas encore toutes abouties, elles témoignent néanmoins du fait que les auteurs de ces ouvrages ont pris conscience de la nécessité de faire entrer les écrivains patrimoniaux et leurs textes en résonance avec le monde d'aujourd'hui : ils sont propices à nous faire réfléchir sur nous-mêmes et sur le monde qui nous entoure. Les nouveaux programmes du cycle 4 , entrés en vigueur à la rentrée 2016, vont dans ce sens en indiquant que la lecture de l'image doit s'inscrire dans le contexte historique et culturel des œuvres. De nombreuses photographies de mises en scène différentes pour une même pièce, dont certaines rendent compte de l'actualité la plus récente, sont présentes dans tous les manuels de 2016 destinés aux élèves du collège. Ainsi, par exemple, dans le manuel des éditions Magnard pour la classe de $5^{\mathrm{e}}$, Jardin des lettres, deux photographies de L'Avare sont proposées à l'observation des élèves : l'une extraite de la mise en scène de Georges Weber, en 2007 au théâtre Toursky de Marseille, avec Michel Bouquet dans le rôle d'Harpagon; l'autre, extraite de la mise en scène de Colette Roumanoff, à Paris, en 2015, avec Laurent Richard ${ }^{20}$. On peut aussi constater un grand enrichissement du corpus iconographique pour inscrire la lecture de l'image dans le contexte historique et culturel des œuvres. Une grande majorité des manuels contextualise donc le cadre de la production de l'œuvre de Molière en proposant des gravures ou photographies de tableaux contemporains du dramaturge. C'est le cas, par exemple, du manuel pour la classe de $6^{\mathrm{e}}$ des éditions Le Robert, dans lequel on peut trouver une Gravure de Jean Le Pautre intitulée Représentation de la pièce du Malade imaginaire, de Molière à la Cour de Versailles, en $1676^{21}$ : l'image aujourd'hui devient ainsi le support privilégié pour inscrire le texte de Molière dans le double jeu de l'actualisation-contextualisation. 


\section{NOTES}

1. O. Marais (dir), Fil d'Ariane, $5^{e}$, Paris, Didier, 2011, p. 225.

2. Ibid., p. 247.

3. N. Combe (dir.), Français, $4^{e}$, Paris, Belin, 2007, p. 182.

4. C. Durand Degranges (dir.), Jardin des lettres, $5^{e}$, Paris, Magnard, 2011, p. 270-275.

5. X. Damas (dir.), Terres littéraires, $2^{\text {nde }}$, Paris, Hatier, 2011, p. 65.

6. A.-M. Bonnabel (dir.), Français, $2^{\text {nde }}$, Paris, Belin, 2010, p. 178-179.

7. J.-D. Ingres, Louis XIV et Molière, 1857, Comédie-Française, Paris.

8. F. Lagache (dir.), L'œil et la plume, $5^{e}$, Paris, Belin, 2011, p. 190.

9. É. Ballanfat (dir.), Jardin des lettres, $6^{e}$, Paris, Magnard, 2013, p. 220-221.

10. Fil d'Ariane, $5^{e}$, ouvr. cité, p. 237.

11. O. Marais (dir.), L'écho des Lettres, Français $2^{\text {nde }}$, Paris, Belin, 2015, p. 156-157.

12. Y. Citton, Lire, interpréter, actualiser. Pourquoi les études littéraires?, Paris, Éditions Amsterdam, 2007, p. 6.

13. L. Carpentier (dir.), Soleils d'encre, Français $1^{\text {re }}$, Paris, Hachette, 2007, p. 185-187.

14. R. Planchon parle ici en son nom et au nom du public contemporain.

15. N. Combe (dir.), Français, $4^{e}$, ouvr. cit., p. 182.

16. F. Lagache (dir.), L'œil et la plume, $4^{e}$, Paris, Belin, 2011, p. 175-188.

17. Ibid.

18. V. Presselin et F. Mouttapas (dir.), L'écume des lettres, $2^{\text {nde }}$, Paris, Hachette, 2011, p. 140-145.

19. Bulletin officiel spécial $\mathrm{n}^{\circ} 9$ du 30 septembre 2010, p. 7.

20. É. Ballanfat (dir.), Jardin des lettres, $5^{e}$, Paris, Magnard, 2016, p. 116.

21. C. Abensour (dir.), Français, $6^{e}$, livre unique, Paris, Le Robert, coll. « Passeurs de textes », 2016, p. 200.

\section{RÉSUMÉS}

L'exemple de Molière et de son théâtre illustre l'effort des auteurs des manuels d'aujourd'hui d'inscrire le dramaturge et son œuvre dans la double thématique de la contextualisationactualisation. L'image de l'auteur est d'abord étudiée avant l'analyse de son œuvre.

L'image de Molière en tant qu'auteur semble avoir un statut particulier qui différencie la représentation du dramaturge de celle de tout autre auteur patrimonial dans la mesure où deux images différentes de Molière coexistent : celle de l'auteur et celle du comédien. Ce qui est aussi unique, c'est que les manuels d'aujourd'hui juxtaposent à ces deux représentations une iconographie qui actualise l'image du dramaturge.

En ce qui concerne l'œuvre de Molière, le double mouvement de l'actualisation-contextualisation par l'image témoigne de l'effort des manuels de permettre à l'élève d'actualiser le contexte de la production des textes de Molière dans une approche qui lui permet de concrétiser le passé à travers des images de notre temps. 
The example of Molière and his theater shows the effort of the authors of textbooks today to include the playwright and his work in the dual theme of contextualisation-discount. The author's image is first examined before the analysis of his work.

The image of Molière as the author seems to have a special status. Two different representations coexist: that of the author and that of the actor. What is also unique, is that contemporary textbooks juxtapose these two representations iconography which updates the image of the playwright.

Regarding the work of Molière, the double movement of the update-contextualization by the image reflects the effort of textbooks allow students to update the context of the production of Molière's texts in approach which enables it to achieve the past through images today.

\section{AUTEUR}

\section{ISABELLE CALLEJA-ROQUE}

ESPE d'Aix-Marseille, Université Grenoble Alpes, UMR 5316 LITT\&ARTS CNRS.

Isabelle Calleja-Roque est professeure agrégée à l'ESPE d'Aix-Marseille. Elle est rattachée à l'UMR 5316 LITT\&ARTS CNRS de l'Université Grenoble Alpes pour sa thèse sur l'évolution de l'image de Molière dans les manuels depuis le XIX siècle, soutenue en décembre 2016. Elle s'intéresse au mythe de l'auteur patrimonial dans l'enseignement de la littérature. 